\title{
A prospective randomised controlled trial of operative versus non-operative management of fractures of the humeral diaphysis: the HUmeral Shaft Fracture FIXation (HU-FIX) Study protocol
}

\author{
William M. Oliver ${ }^{*}(\mathbb{D}$, Thomas H. Carter, Catriona Graham, Timothy O. White, Nicholas D. Clement,
} Andrew D. Duckworth and Samuel G. Molyneux

\begin{abstract}
Background: Humeral shaft fractures constitute around $1 \%$ of adult fractures in the UK, with an annual incidence of approximately 13 per 100,000 population. Historically, these injuries have been primarily managed non-operatively, with operative fixation reserved for specific indications. Although some recent retrospective studies have suggested there are potential benefits of operative fixation over humeral bracing, there is a deficiency in level 1 evidence to support operative management as the primary treatment for humeral shaft fractures.

Methods/design: This single-centre prospective randomised controlled trial aims to recruit 70 adult patients with an isolated closed fracture of the humeral diaphysis into one of two treatment arms: operative $(n=35)$ or non-operative $(n=35)$. The operative arm will undergo open reduction and internal fixation (ORIF) of the fracture using a standard fixation technique (plate and screws). The non-operative arm will be fitted with a prefabricated humeral brace until fracture union. All patients will be followed up for 1 year post-intervention. The primary outcome measure will be the Disabilities of the Arm, Shoulder and Hand (DASH) score at 3 months post-intervention. Secondary outcome measures will include pain, treatment complications, return to work or sporting activities, shoulder and elbow range of motion, radiographic assessment, EuroQol (EQ-5D) Health Outcome score and 12-item Short Form (SF-12) Health Survey score. A health economic analysis will be performed to compare the cost implications of each treatment strategy.
\end{abstract}

Discussion: This randomised controlled trial will provide level 1 evidence comparing a standard ORIF technique against functional bracing for isolated closed humeral shaft fractures. The investigators hope that the study results will assist surgeons in their decision-making when managing patients with these injuries.

Trial registration: ClinicalTrials.gov, NCT03689335. Registered on 28 September 2018 (retrospectively).

Keywords: Humerus, Humeral, Shaft, Diaphysis, Fracture, Randomised controlled trial, Operative, Fixation, Nonoperative, Bracing, Trauma, Patient outcome

\footnotetext{
* Correspondence: william.m.oliver@doctors.org.uk

Edinburgh Orthopaedic Trauma, Royal Infirmary of Edinburgh, 51 Little

France Crescent, Edinburgh, Midlothian EH16 4SA, UK
}

(c) The Author(s). 2019 Open Access This article is distributed under the terms of the Creative Commons Attribution 4.0 International License (http://creativecommons.org/licenses/by/4.0/), which permits unrestricted use, distribution, and reproduction in any medium, provided you give appropriate credit to the original author(s) and the source, provide a link to the Creative Commons license, and indicate if changes were made. The Creative Commons Public Domain Dedication waiver (http://creativecommons.org/publicdomain/zero/1.0/) applies to the data made available in this article, unless otherwise stated. 


\section{Background}

Fractures of the humeral diaphysis (humeral shaft fractures) constitute $1.2 \%$ of adult fractures in the UK, with an overall incidence of 12.9 per 100,000 per year [1]. These injuries are more common in women and have a bimodal age distribution, with peaks of prevalence occurring in younger adults following high-energy trauma and older adults following low-energy trauma [1-3].

Humeral shaft fractures are routinely managed nonoperatively, with the widespread use of a prefabricated humeral brace, as first described by Sarmiento et al. in 1977 [4]. The purported benefits of this technique (over conventional plaster immobilisation) include rapid and uninterrupted osteogenesis and early mobilisation of the shoulder and elbow, thus enhancing functional outcomes [4]. Subsequent studies comparing bracing with plaster immobilisation have demonstrated faster fracture union, reduced rates of varus mal-alignment and confirmed functional advantages in terms of shoulder and elbow mobility $[5,6]$.

Although functional bracing has resulted in low rates of humeral shaft fracture nonunion in some series [7-9], others have reported nonunion rates of $10 \%$ or more [10-13], with the highest rate reported as 33\% [14]. When fracture union does occur, the reported time to union ranges from 7.5 to 11.5 weeks $[7,9]$, during which time almost all activities of daily living are restricted, and there is a risk of skin breakdown and secondary cellulitis that can necessitate brace removal [13]. Moreover, while malunion is considered to be tolerated well by patients, the acceptable thresholds are based upon an historic series of just 32 patients, who were examined by a single author [15]. A more recent series has suggested that malunion and consequent loss of shoulder range of motion can occur in up to $38 \%$ of patients treated with a brace [16].

The perceived limitations of humeral bracing have brought about a gradually increasing role for operative fixation [17]. Although intramedullary nailing is considered feasible, published case series [18], reviews [19, $20]$ and randomised controlled trials [21, 22] have consistently recommended open reduction and internal fixation (ORIF) as the strategy of choice when surgery is indicated.

The primary aim of this trial is to determine whether any functional difference exists, according to the Disabilities of the Arm, Shoulder and Hand (DASH) score at 3 months post-intervention, between operative (ORIF) and non-operative management (functional bracing) of humeral shaft fractures. The secondary aim of this trial is to determine whether any difference exists in other clinically important outcomes (including pain, treatment complications, return to work or sporting activities, shoulder and elbow range of motion, fracture union, patient-reported outcome measures and economic costs) between ORIF and functional bracing of humeral shaft fractures in the year following the intervention.

\section{Methods/design}

The Humeral Shaft Fracture Fixation (HU-FIX) Study is a single-centre prospective randomised controlled trial that aims to assess whether there is any difference in outcome between patients with a humeral shaft fracture who are managed operatively (i.e. with surgical fixation) and those managed non-operatively. The study will adhere to the principles in the latest Consolidated Standards of Reporting Trials (CONSORT) Statement [23]. The study will be performed at a large tertiary referral centre. The study received a favourable opinion from the relevant research ethics committee on 19 July 2018 (South-East Scotland Research Ethics Committee 01 reference 18/SS/0073). The study is sponsored by National Health Service (NHS) Lothian Research and Development and was approved on 10 September 2018 (project number 2018/0223). The study was registered with the ClinicalTrials.gov database, which is operated by the US National Library of Medicine, on 28 September 2018 (ID NCT03689335). The study is funded and co-sponsored by the Scottish Orthopaedic Research Trust into Trauma (SORT-iT).

The trial will include all eligible patients presenting to the study centre with an isolated closed fracture of the humeral shaft. The planned flow of patients through the study is as shown in Fig. 1.

A fracture of the humeral shaft (diaphyseal segment) is defined as any humeral fracture in which the major fracture line does not extend to within one metaphyseal width (Müller box) of either the shoulder or elbow joint; this is consistent with the Arbeitsgemeinschaft für Osteosynthesefragen definition [24], and is illustrated in Fig. 2.

\section{Inclusion criteria}

1. Fracture of the humeral shaft

2. Closed injury

3. Age $\geq 16$ years

4. Able to provide informed consent in English

5. Surgery performed within 3 weeks of date of injury

\section{Exclusion criteria}

1. Completely undisplaced fractures

2. Injuries considered to be an absolute indication for surgery (including severe associated neurovascular injury, open fractures and bilateral injuries)

3. Patients with a periprosthetic or pathological fracture 


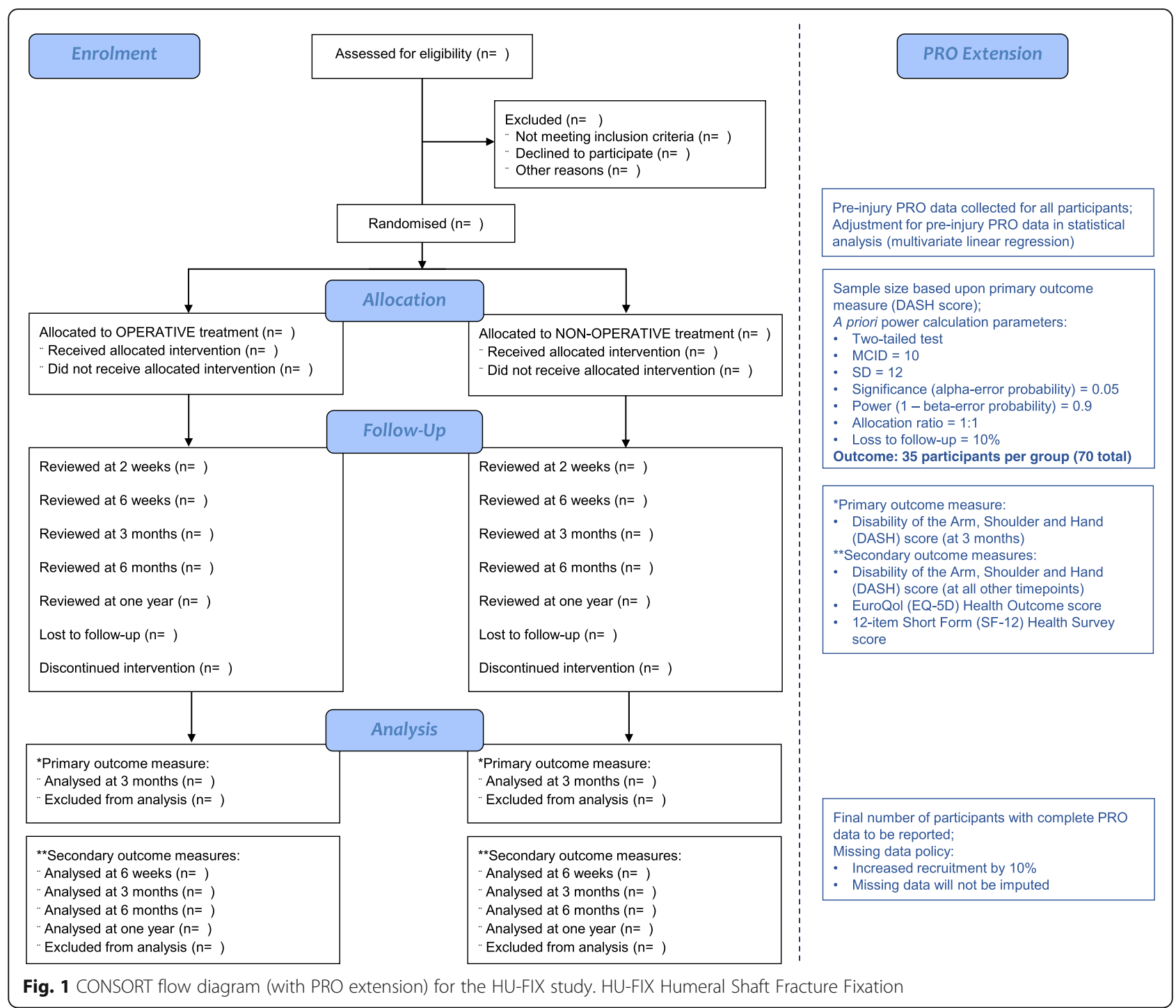

4. Patients with an additional spine or limb injury (including those with polytrauma), which may impact upon functional rehabilitation

5. Patients medically unfit for surgery

6. Very frail patients, defined as those with a Clinical Frailty Score of $\geq 6 / 9$ as described in the Canadian Study on Health and Aging [25]

7. Pregnant women with predetermined treatment

8. Patients declining operative management

9. Patients unable to comply with post-operative data gathering, including completing questionnaires in English

10. Non-residents or those unable to return to the Unit for the 1-year follow-up period

11. Patients for whom the treating surgeon does not feel that inclusion in the trial is in their best interests, either due to fracture pattern or patient factors

\section{Enrolment and allocation}

Potential HU-FIX study participants will be identified at the point of referral to the Orthopaedic Service, either directly (via the on-call team) or via the Trauma Triage Clinic system [26]. Patients satisfying the inclusion and exclusion criteria will be introduced to the study by the treating clinical team and provided with a patient information sheet to read before being asked to participate. Patients will be predominantly enrolled into the study either on the Orthopaedic Ward (if admitted) or at their first outpatient clinic appointment. A study investigator will review the study protocol in detail with the patient and address any of their questions. If the patient is willing to participate, a study investigator will complete the informed consent process.

Once informed consent has been obtained, patients will be enrolled into the study by an investigator and 


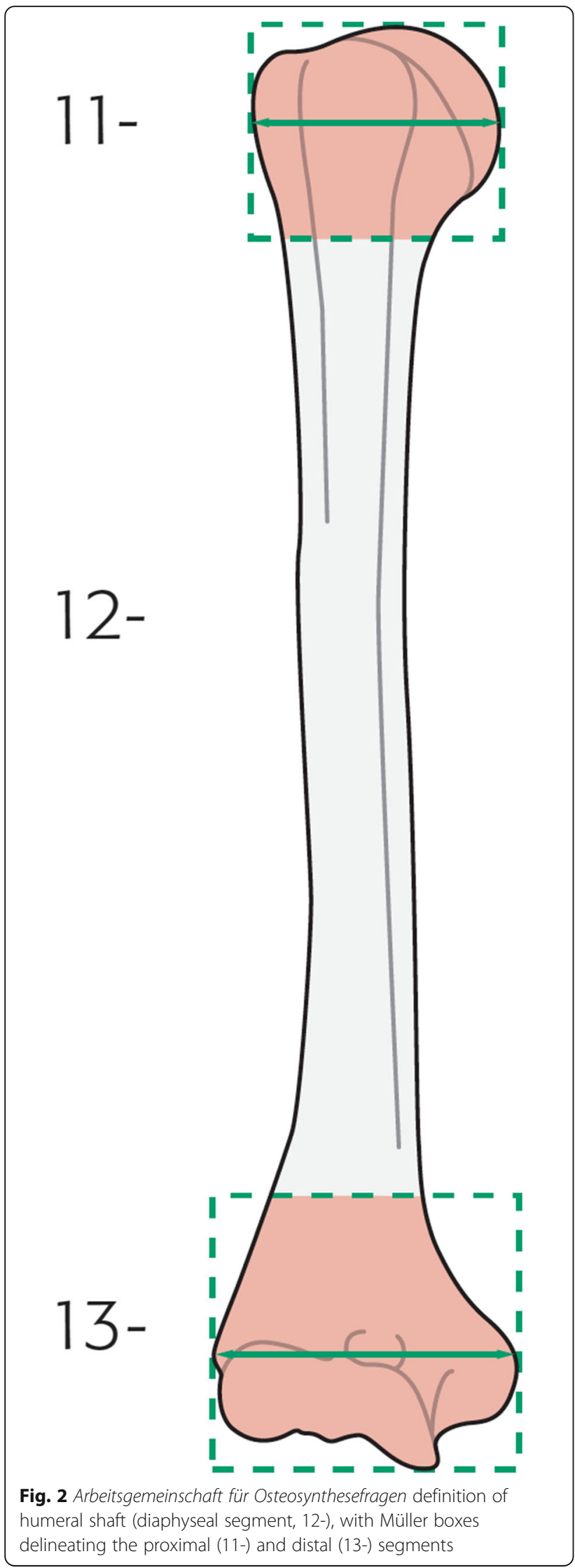

randomly allocated to either operative or non-operative management of their humeral shaft fracture.

\section{Randomisation}

The study will use a parallel assignment model, with 1:1 allocation of patients into each treatment arm (operative or non-operative). Randomisation will be stratified by patient age $(<65$ years old or $\geq 65$ years old), to ensure there are approximately equal numbers of younger and older patients in each treatment arm. Using block randomisation with a mixed block size and a 1:1 ratio of operative to non-operative, a computer-generated randomisation schedule was produced by a senior statistician from the local clinical research facility using nQuery Advisor v7.0 software (Statsols, Cork, Ireland). A member of staff independent of the study used this schedule to produce a series of opaque sealed envelopes, each containing a sticker bearing the words 'operative' or 'non-operative'. Once the randomisation envelope is opened, the sticker is placed onto the already-signed consent form to indicate the treatment group allocation.

\section{Interventions}

All patients will be initially treated in the Emergency Department with closed reduction of their humeral shaft fracture, and application of a either a U-slab or aboveelbow hanging plaster cast.

Patients allocated to operative management of their humeral shaft fracture will undergo surgical fixation, using a standard technique of plating and screw fixation. The exact surgical approach and fixation technique utilised will be at the discretion of the treating surgeon. Similarly, post-operative immobilisation and range-ofmotion restrictions will be at the discretion of the treating surgeon. This is determined by a number of factors including the injury and fracture pattern, bone quality, co-morbidities and patient compliance. The approach, fixation technique and range-of-motion restrictions will be recorded for all patients and presented as a subgroup analysis. Although the study sample will not be specifically powered on this basis (see Power calculation and statistical analysis, below), the investigators hope that a sub-group analysis will nonetheless allow any differences between the groups to be detected.

Patients allocated to non-operative management will be managed as per current standard practice for the study centre. This involves a period of immobilisation in a U-slab or hanging cast for up to 2 weeks, before fitting with a lightweight prefabricated humeral brace in the outpatient clinic. Two specific models of humeral brace are in routine use in this centre: the Clasby humeral brace (Beagle Orthopaedic, Blackburn, UK) and the ProCare over-the-shoulder humeral fracture brace (DJO Global, Vista, CA). The current standard of care in the 
study centre is to use the Clasby brace for proximalthird or mid-shaft fractures and the ProCare brace for distal-third or multifragmentary fractures. Brace selection for study participants will reflect these indications but will remain at the treating surgeon's discretion. Patients will be permitted to start passive pendular shoulder exercises with full elbow, wrist and hand mobilisation as soon as the humeral brace has been applied. Post-injury physiotherapy will be arranged at the discretion of the treating surgeon, as occurs in everyday clinical practice.

\section{Outcome assessment}

All follow-up assessments will take place during outpatient clinic visits at 2 weeks, 6 weeks, 3 months, 6 months and 1 year. At each clinic visit, a physical examination will be performed, complications noted and the need for any further surgery recorded. Patients will be given time to complete a self-reported questionnaire, which will generate clinical outcome scores. The 1-year follow-up will enable study investigators to assess whether any differences between the operative and non-operative groups (if any) are sustained for 1 year post-intervention and to detect any important complications that may occur within the first year (e.g. nonunion).

The Standard Protocol Items: Recommendations for Interventional Trials (SPIRIT) [27] checklist is given in Additional file 1. The SPIRIT schedule of enrolment, interventions and assessments is shown in Fig. 3.

\section{Primary outcome measure}

The primary outcome measure is the DASH score at 3 months post-intervention. The DASH score is a functional rating scale that was developed in 1996. It has been used extensively as a research tool in upper limb surgery [28]. It consists of a 30-item self-reported questionnaire, and two optional modules, one assessing work and the other sports and performing arts. Patient symptoms (including pain, weakness, stiffness and tingling or numbness) and functional status (including physical, social and psychological aspects) during the week prior to survey completion are assessed. The final score is from 0 to 100 , with 0 representing no disability and 100 the worst possible disability. A recent study demonstrated the DASH score to be both valid and reliable in evaluating outcomes in patients following a humeral shaft fracture [29].

The null hypothesis is that there is no difference in early functional outcomes (DASH score at 3 months post-intervention) between the operative and non-operative management of patients following a humeral shaft fracture.

\section{Secondary outcome measures}

Secondary outcome measures will include:

1. Change in the DASH score over the 1-year follow-up period

2. Pain assessment, using a visual analogue scale (0 to 10)

3. Complications of treatment, including neurovascular injury, superficial or deep infection, failure of fixation, revision surgery, skin complications (including eczema and cellulitis) and complex regional pain syndrome

4. Time to return to work and sports (if relevant)

5. Satisfaction with the service, using a visual analogue scale (0 to 100), and with the appearance of the affected limb, also using a visual analogue scale (0 to 10 )

6. Range of motion at the shoulder and elbow

7. Radiographic assessment, using standard anteroposterior and lateral radiographs of the humerus to assess the progression of fracture healing (union or nonunion) and final humeral deformity (malunion)

8. EuroQol (EQ-5D) health outcome score [30]

9. 12-item Short Form (SF-12) health survey score [31]

10. Health economic analysis

Outcome measures 1 to 9 have been collated into a single data collection form (one form per study participant).

\section{Power calculation and statistical analysis}

Prior to study commencement, a prospective power calculation was used to determine the number of patients required in each treatment group. The minimal clinically important difference in the DASH score is reported to be 10 points [32], and the assumed standard deviation is 12 points for both the operative and non-operative groups [33]. Thus, using a two-sided $t$-test with $5 \%$ significance, a total of 70 patients (35 in each group) are required to produce a $90 \%$ power to detect a meaningful difference (in terms of the DASH score at 3 months post-intervention) between the two groups, assuming a $10 \%$ loss to follow-up.

Data will be analysed on an intention-to-treat basis. Data analysis will be performed by a senior statistician, employed by the local clinical research facility and independent of the study team. The primary outcome measure (DASH score at 3 months post-intervention) will be compared between the two treatment arms, using a twosided independent samples $t$-test or non-parametric equivalent (as indicated by the normality of the data). This method will also be used to compare the other continuous outcome measures between the two treatment arms (e.g. pain score, satisfaction scores, shoulder and 


\begin{tabular}{|c|c|c|c|c|c|c|c|}
\hline \multirow[b]{3}{*}{ TIMEPOINT (weeks) } & \multicolumn{7}{|c|}{ STUDY PERIOD } \\
\hline & \multirow{2}{*}{$\frac{\text { Enrolment }}{-}$} & \multirow{2}{*}{$\begin{array}{c}\text { Intervention } \\
0\end{array}$} & \multicolumn{4}{|c|}{ Post-intervention } & \multirow{2}{*}{$\frac{\text { Close-out }}{52}$} \\
\hline & & & 2 & 6 & 12 & 26 & \\
\hline \multicolumn{8}{|l|}{ ENROLMENT: } \\
\hline Eligibility screen & $x$ & & & & & & \\
\hline Informed consent & $x$ & & & & & & \\
\hline Demographics & $x$ & & & & & & \\
\hline Injury details & $x$ & & & & & & \\
\hline Allocation & $x$ & & & & & & \\
\hline \multicolumn{8}{|l|}{ INTERVENTIONS: } \\
\hline Non-operative & & $x$ & & & & & \\
\hline Operative & & $x$ & & & & & \\
\hline \multicolumn{8}{|l|}{ ASSESSMENTS: } \\
\hline Radiographs & $x$ & $x$ & $x$ & $x$ & $x$ & & $x$ \\
\hline DASH score & $x$ & & & $x$ & $x$ & $x$ & $x$ \\
\hline SF-12 score & $x$ & & & $x$ & $x$ & $\mathrm{X}$ & $x$ \\
\hline$E Q-5 D$ score & $x$ & & & $x$ & $x$ & $x$ & $x$ \\
\hline Pain VAS & & & $x$ & $x$ & $x$ & $x$ & $x$ \\
\hline Complications & & & $x$ & $x$ & $x$ & $\mathrm{x}$ & $x$ \\
\hline Return to work/sport & & & $x$ & $x$ & $x$ & $x$ & $x$ \\
\hline Satisfaction VAS & & & $x$ & $x$ & $x$ & $x$ & $x$ \\
\hline Shoulder/elbow ROM & & & & $x$ & $x$ & $x$ & $x$ \\
\hline \multicolumn{8}{|c|}{$\begin{array}{l}\text { DASH, Disabilities of Arm, Shoulder and Hand; SF-12, 12-item Short Form health survey; EQ-5D, } \\
\text { EuroQol health outcome; VAS, visual analogue scale; ROM, range of motion }\end{array}$} \\
\hline \multicolumn{8}{|c|}{$\begin{array}{l}\text { Fig. } 3 \text { SPIRIT figure for the HU-FIX study. DASH Disabilities of the Arm, Shoulder and Hand, EQ-5D EuroQol Health Outcome, ROM range of } \\
\text { movement, SF-12 12-item Short Form Health Survey, VAS visual analogue scale }\end{array}$} \\
\hline
\end{tabular}


elbow range of motion, EQ-5D score and SF-12 score). The pattern of change in continuous outcome measures over the study period will be presented graphically, broken down by treatment allocation. A linear regression model will be generated for each participant by plotting the DASH score from 6 weeks to 1 year postintervention and the gradient of the regression line (i.e. the rate of change in the score) will be compared between treatment groups. Multivariate linear regression, including analysis of covariance (ANCOVA), will also be used to adjust for the potential effect of pre-injury DASH score, age and other prognostic factors affecting the DASH score. Binary outcomes (e.g. treatment complications, re-operation and nonunion or malunion) will be compared between the two treatment arms using a binomial test for the comparison of proportions. Twotailed $p$ values will be reported where possible, and statistical significance will be set at $p=0.05$.

\section{Missing data}

The study sample size has been increased to provide for up to $10 \%$ loss to follow-up at the primary outcome timepoint (3 months post-intervention). All clinical and outcome data will be collected in the outpatient clinic, where clinical reviews and radiographs (where required) are also performed. These appointments reflect routine follow-ups of a humeral shaft fracture in the study centre, and the investigators anticipate this arrangement will limit missing data considerably. The participant dropout rate for each treatment arm will be reported and compared. If the participant dropout rate at the primary outcome timepoint is substantial, a sensitivity analysis may be performed. Missing data at other timepoints will be accounted for by plotting a line of best fit for the data points for each participant and comparing the gradient of the regression line (i.e. the rate of change in the variable of interest) between treatment groups.

\section{Health economic analysis}

Direct costs of the injury and its treatment will be assessed, including the costs for any of the following:

- plaster or brace immobilisation

- any surgical procedure (including implant costs, theatre time, hospital admission and treatment of early complications)

- analgesia or other treatments for ongoing pain or other complications (pharmaceutical or otherwise)

- any additional clinical input (including primary and secondary care encounters and re-admissions to hospital)

- any allied health professional input (including physiotherapy and occupational therapy)
- any occupational or statutory sick pay received during employment absence

Secondary costs will also be assessed, including:

- any loss of earnings

- any additional support required for patients to complete their activities of daily living or to fulfil their own caring responsibilities to others

These data will allow an analysis of the overall economic impact of humeral shaft fractures and a comparison of the cost implications of each treatment strategy. A cost per quality-adjusted life year (QALY) gained will be calculated (total cost difference between treatment groups / difference in QALYs between treatment groups). QALYs will be calculated at 1 year, using the difference between the EQ-5D scores at the followup intervals.

\section{Patient involvement, safety and confidentiality}

Patient and public involvement was not sought for this small, single-centre trial.

The maximum possible radiation dose for study participants is equivalent to 2 weeks of the dose to the UK population from natural sources of background radiation. The risk of fatal cancer arising from this level of exposure is negligible, at 1 in 230,000 [34]. This proposed radiation exposure has been approved by a medical physics expert and a consultant radiologist.

Complication rates will be monitored by study investigators throughout the trial, but a formal data monitoring committee will not be convened. Both treatment options (operative and non-operative) are regularly employed in the study centre, and any additional risk to patient safety is low. The study sponsor will be regularly updated with study progress and any patient safety issues that may arise, including adverse or serious adverse events. The trial steering committee will be consulted if required. The procedure for identifying, recording and reporting adverse events and urgent safety measures will adhere to relevant guidelines from the study sponsor, which will also advise upon any action required to protect patient safety.

Data collection forms will be kept in a secure filing cabinet in the research office on the study site, both of which are locked when not in use by the study investigators. These personal data will then be entered and stored electronically, under a password-protected file on a password-protected NHS computer system and will be accessible only by the study investigators. Once data collection is complete and analysis begins, non-personal data (stored using unique participant numbers rather than names or other identifiable information) will be 
made available to other authorised members of the research team. All electronic data will be handled according to study sponsor guidelines on data protection and confidentiality.

When finalised, study results will be disseminated via an internal report, conference presentations and peerreviewed scientific journals. A summary of the results will be made available to study participants upon request.

\section{Discussion}

A review of the existing literature indicates that there is a paucity of level 1 evidence to support operative management as the primary treatment for humeral shaft fractures. Several studies have retrospectively compared humeral shaft fracture outcomes following non-operative and operative management. Jawa et al. compared complication rates for distal-third humeral shaft fractures treated with bracing versus those treated with ORIF, concluding that both strategies had potential risks and that the optimal treatment was based on patient preference [35]. More recently, Denard et al. demonstrated that there was an increased likelihood of both nonunion and malunion with bracing, and in the context of comparable (or lower) complication rates with ORIF, concluded that ORIF should be performed more frequently [36]. Mahabier et al. also suggested there was little difference between non-operative and operative treatment in terms of fracture union or nerve injury [37].

In the only published randomised controlled trial of which the authors are aware, Matsunaga et al. compared bracing with a less widely employed minimally invasive bridge plating technique, demonstrating a statistically (but not clinically) significant difference in the DASH score in favour of the fixation group, along with significantly lower rates of nonunion, contact dermatitis and coronal plane malalignment at 6 months in the fixation group [38]. There was no significant difference in the DASH score at any other timepoint, and no difference in any other functional outcomes between the groups. However, this study did not assess the current gold standard surgical technique of compression plating and included a population substantially younger than the average patient sustaining a humeral shaft fracture in the UK [1].

Two ongoing randomised controlled trials comparing surgical fixation against functional bracing are currently recruiting patients, one in Canada (ClinicalTrials.gov ID NCT00878319) and one in Finland (ClinicalTrials.gov ID NCT01719887) [39]. A further prospective multicentre observational study comparing functional recovery after operative versus non-operative treatment is also underway in the Netherlands (Netherlands Trial Register
ID NTR3617) [40]. The HU-FIX study aims to contribute to this growing body of literature and provide greater clarity to surgeons managing patients with humeral shaft fractures.

The study is pragmatic and allows for normal variations in clinical practice, including in the surgical approach and fixation method used during ORIF, as well as the involvement of physiotherapy. The investigators hope that this pragmatic design will reproduce day-to-day trauma care and improve the external validity of study results [41, 42]. Potential limitations include the single-centre study design and the lack of blinding. Edinburgh Orthopaedic Trauma provides trauma care to a catchment population of approximately 850,000 and consists of 12 consultant orthopaedic trauma surgeons who collectively treat approximately 70 humeral shaft fractures per year [1]. The investigators are, therefore, confident that the study results can be reliably extrapolated to the wider orthopaedic community. Patient blinding is not possible in this study given the nature of the interventions being compared, neither is assessor blinding, as the presence or absence of a surgical scar or metalwork on plain radiographs will be obvious during outcome assessment. Again, this is consistent with a pragmatic design that reflects everyday clinical practice. Although this study is not a pilot for a larger multi-centre randomised controlled trial, the investigators hope that the results of the HU-FIX Study will inform researchers planning or preparing a multicentre trial in the future.

Use of patient-reported measures to assess outcomes after a traumatic injury is consistently expanding [43], with the main advantage being that determining injury outcome or treatment success is increasingly becoming patient-centred. The DASH score is a holistic measure of outcome, assessing impairment, activity limitation and participant restriction [44]. However, it does not distinguish between the injured and uninjured limbs, which may artificially inflate scores for patients who are more able to compensate for the loss of function in one limb (e.g. young active patients with injuries to their non-dominant arm). It is, therefore, important to adjust for these potential confounding factors during statistical analysis.

\section{Trial status}

This manuscript is based on HU-FIX study protocol version 1.3 (29 August 2018). The first patient was enrolled on 19 September 2018. The investigators estimate enrolment will be complete by July 2020 , with the study follow-up complete by July 2021. 


\section{Additional file}

Additional file 1: SPIRIT 2013 Checklist: Recommended items to address in a clinical trial protocol and related documents. (DOC $127 \mathrm{~kb}$ )

\section{Abbreviations}

CONSORT: Consolidated Standards of Reporting Trials; DASH: Disabilities of the Arm, Shoulder and Hand; EQ-5D: EuroQol Health Outcome; HU-FIX: Humeral Shaft Fracture Fixation; NHS: National Health Service; ORIF: Open reduction and internal fixation; QALY: Quality-adjusted life year; ROM: Range of movement; SF-12: 12item Short Form Health Survey; SORT-iT : Scottish Orthopaedic Research Trust into Trauma; SPIRIT: Standard Protocol Items: Recommendations for Interventional Trials; VAS: Visual analogue scale

\section{Acknowledgements}

This study is being conducted with the support of SORT-iT and NHS Lothian Health Board. The authors would like to thank Peter Hall, Fergus Perks, Kenny Scott and Christina Stewart for their expert advice; the Emergency Medicine Research Group of Edinburgh for their advice and assistance in patient recruitment; and Deborah Ketchen and Keri Swain for their assistance in study administration.

\section{Authors' contributions}

SGM primarily conceived of the study, contributed to development of the protocol and is the principal study investigator. WMO contributed to study design, developed the protocol, drafted the manuscript and acts as the main co-ordinating study investigator. ADD, NDC and TOW contributed to study design and development of the protocol, and act as study co-investigators. THC assisted with manuscript preparation and acts as an additional study investigator. CG contributed to study design and provided detailed statistical guidance. All authors have contributed to and approved the final manuscript.

\section{Authors' information}

WMO is enrolled with the University of Edinburgh in a postgraduate doctor of medicine (MD) degree programme and this study will form part of his MD thesis submission. All authors have valid good clinical practice qualifications.

\section{Funding}

The study is funded by SORT-iT.

\section{Availability of data and materials}

Study data will be made available upon request.

\section{Ethics approval and consent to participate}

The study has been approved by South-East Scotland Research Ethics Committee 01 (reference 18/SS/0073). Informed consent will be obtained from all participants prior to enrolment into the study.

\section{Consent for publication}

Not applicable.

\section{Competing interests}

The authors declare that they have no competing interests.

Received: 27 October 2018 Accepted: 16 July 2019

Published online: 05 August 2019

\section{References}

1. Court-Brown CM, Caesar B. Epidemiology of adult fractures: A review. Injury. 2006:37(8):691-7.

2. Ekholm R, Adami J, Tidermark J, Hansson K, Tornkvist H, Ponzer S. Fractures of the shaft of the humerus: An epidemiological study of 401 fractures. $J$ Bone Joint Surg Br. 2006;88-B(11):1469-73.

3. Bergdahl C, Ekholm C, Wennergren D, Nilsson F, Möller M. Epidemiology and patho-anatomical pattern of 2,011 humeral fractures: Data from the Swedish Fracture Register. BMC Musculoskelet Disord. 2016;17(1):1-10.

4. Sarmiento A, Kinman PB, Galvin EG, Schmitt RH, Phillips JG. Functional bracing of fractures of the shaft of the humerus. J Bone Joint Surg Am. 1977:59(5):596-601.
5. Sharma VK, Jain AK, Gupta RK, Tyagi AK, Sethi PK. Non-operative treatment of fractures of the humeral shaft: a comparative study. J Indian Med Assoc. 1991;89(6):157-60

6. Camden P, Nade S. Fracture bracing the humerus. Injury. 1992;23(4):245-8.

7. Balfour GW, Mooney $V$, Ashby ME. Diaphyseal fractures of the humerus treated with a ready-made fracture brace. J Bone Joint Surg Am. 1982; 64(1):11-3.

8. Zagorski JB, Latta LL, Zych GA, Finnieston AR. Diaphyseal fractures of the humerus. Treatment with prefabricated braces. J Bone Joint Surg Am. 1988; 70(4):607-10.

9. Sarmiento A, Zagorski JB, Zych GA, Latta LL, Capps CA. Functional bracing for the treatment of fractures of the humeral diaphysis. J Bone Joint Surg Am. 2000:82(4):478-86.

10. Naver L, Aalberg JR. Humeral shaft fractures treated with a ready-made fracture brace. Arch Orthop Trauma Surg. 1986;106(1):20-2.

11. Koch PP, Gross DFL, Gerber C. The results of functional (Sarmiento) bracing of humeral shaft fractures. J Shoulder Elb Surg. 2002;11(2): $143-50$.

12. Toivanen JAK, Nieminen J, Laine H-J, Honkonen SE, Järvinen MJ. Functional treatment of closed humeral shaft fractures. Int Orthop. 2005;29(1):10-3.

13. Rutgers $M$, Ring $D$. Treatment of diaphyseal fractures of the humerus using a functional brace. J Orthop Trauma. 2006;20(9):597-601.

14. Harkin FE, Large RJ. Humeral shaft fractures: union outcomes in a large cohort. J Shoulder Elb Surg. 2017;26(11):1881-8.

15. Klenerman $L$. Fractures of the shaft of the humerus. J Bone Joint Surg Br. 1966;48(1):105-11.

16. Fjalestad T, Strømsøe K, Salvesen P, Rostad B. Functional results of braced humeral diaphyseal fractures: why do $38 \%$ lose external rotation of the shoulder? Arch Orthop Trauma Surg. 2000;120(5-6):281-5.

17. Schoch BS, Padegimas EM, Maltenfort M, Krieg J, Namdari S. Humeral shaft fractures: national trends in management. J Orthop Traumatol. 2017:18(3):259-63.

18. Bell MJ, Beauchamp CG, Kellam JK, McMurtry RY. The results of plating humeral shaft fractures in patients with multiple injuries. The Sunnybrook experience. J Bone Joint Surg Br. 1985;67(2):293-6.

19. Gregory PR, Sanders RW. Compression Plating Versus Intramedullary Fixation of Humeral Shaft Fractures. J Am Acad Orthop Surg. 1997;5(4):215-23.

20. Bhandari M, Devereaux PJ, McKee MD, Schemitsch EH. Compression plating versus intramedullary nailing of humeral shaft fractures-a meta-analysis. Acta Orthop. 2006:77(2):279-84

21. Chapman JR, Henley MB, Agel J, Benca PJ. Randomized prospective study of humeral shaft fracture fixation: intramedullary nails versus plates. J Orthop Trauma. 2000;14(3):162-6.

22. McCormack RG, Brien D, Buckley RE, McKee MD, Powell J, Schemitsch EH. Fixation of fractures of the shaft of the humerus by dynamic compression plate or intramedullary nail. J Bone Joint Surg Br. 2000;82(3):336-9.

23. Schulz KF, Altman DG, Moher D, CONSORT Group. CONSORT 2010 Statement: updated guidelines for reporting parallel group randomised trials. Trials. 2010;11(1):32

24. Kellam J, Audigé L. Fracture Classification. In: Rüedi T, Buckley R, Moran C, editors. AO Principles of Fracture Management: Volume 1 - Principles. Stuttgart: Thieme; 2007.

25. Rockwood K, Song X, MacKnight C, Bergman H, Hogan DB, McDowell I, et al. A global clinical measure of fitness and frailty in elderly people. Can Med Assoc J. 2005:173(5):489-95.

26. White TO, Mackenzie SP, Carter TH, Jefferies JG, Prescott OR, Duckworth AD, et al. The evolution of fracture clinic design. Bone Joint J. 2017:99B(4):503-7.

27. Chan AW, Tetzlaff JM, Gotzsche PC, Altman DG, Mann H, Berlin JA, et al. SPIRIT 2013 explanation and elaboration: guidance for protocols of clinical trials. BMJ. 2013;346:e7586.

28. Hudak PL, Amadio PC, Bombardier C, Beaton D, Cole D, Davis A, et al. Development of an upper extremity outcome measure: the DASH (disabilities of the arm, shoulder and hand) [corrected]. The Upper Extremity Collaborative Group (UECG). Am J Ind Med. 1996:29(6):602-8.

29. Mahabier KC, Den Hartog D, Theyskens N, Verhofstad MHJ, Van Lieshout EMM. HUMMER Trial Investigators. Reliability, validity, responsiveness, and minimal important change of the Disablities of the Arm, Shoulder and Hand and Constant-Murley scores in patients with a humeral shaft fracture. J Shoulder Elb Surg. 2017;26(1):e1-12.

30. EuroOol Group. EuroOol - a new facility for the measurement of healthrelated quality of life. Health Policy. 1990;16(3):199-208. 
31. Ware J, Kosinski M, Keller SD. A 12-Item Short-Form Health Survey: construction of scales and preliminary tests of reliability and validity. Med Care. 1996;34(3):220-33.

32. Franchignoni F, Vercelli S, Giordano A, Sartorio F, Bravini E, Ferriero G. Minimal clinically important difference of the disabilities of the arm, shoulder and hand outcome measure (DASH) and its shortened version (QuickDASH). J Orthop Sports Phys Ther. 2014;44(1):30-9.

33. The DASH and QuickDASH Outcome Measure User's Manual (Third Edition). 2018. Available from: http://www.dash.iwh.on.ca/dash-manual. Cited 3 Feb 2018.

34. Martin CJ. Effective dose: how should it be applied to medical exposures? Br J Radiol. 2007;80(956):639-47.

35. Jawa A, McCarty P, Doornberg J, Harris M, Ring D. Extra-articular distal-third diaphyseal fractures of the humerus. A comparison of functional bracing and plate fixation. J Bone Jointt Surg Am. 2006;88(11):2343-7.

36. Denard AJ, Richards JE, Obremskey WT, Tucker MC, Floyd M, Herzog GA. Outcome of nonoperative vs operative treatment of humeral shaft fractures: a retrospective study of 213 patients. Orthopedics. 2010;33(8). https://doi. org/10.3928/01477447-20100625-16.

37. Mahabier KC, Vogels LMM, Punt BJ, Roukema GR, Patka P, Van Lieshout EMM. Humeral shaft fractures: Retrospective results of non-operative and operative treatment of 186 patients. Injury. 2013:44(4):427-30.

38. Matsunaga FT, Tamaoki MJS, Matsumoto MH, Netto NA, Faloppa F, Belloti $J C$. Minimally invasive osteosynthesis with a bridge plate versus a functional brace for humeral shaft fractures: A randomized controlled trial. J Bone Joint Surg Am. 2017;99(7):583-92.

39. Rämö L, Taimela S, Lepola V, Malmivaara A, Lähdeoja T, Paavola M. Open reduction and internal fixation of humeral shaft fractures versus conservative treatment with a functional brace: A study protocol of a randomised controlled trial embedded in a cohort. BMJ Open. 2017;7(7): e014076.

40. Mahabier KC, Van Lieshout EMM, Bolhuis HW, Bos PK, Bronkhorst MWGA, Bruijninckx MMM, et al. HUMeral Shaft Fractures: MEasuring Recovery after Operative versus Non-operative Treatment (HUMMER): A multicenter comparative observational study. BMC Musculoskelet Disord. 2014;15:39.

41. Schwartz D, Lellouch J. Explanatory and pragmatic attitudes in therapeutical trials. J Clin Epidemiol. 2009:62(5):499-505.

42. Costa ML, Achten J, Parsons NR, Rangan A, Griffin D, Tubeuf S, et al. Percutaneous fixation with Kirschner wires versus volar locking plate fixation in adults with dorsally displaced fracture of distal radius: randomised controlled trial. BMJ. 2014;349:94807.

43. Rosenberg GM, Stave C, Spain DA, Weiser TG. Patient-reported outcomes in trauma: A scoping study of published research. Trauma Surg Acute Care Open. 2018:3(1):1-6.

44. Dixon D, Johnston M, McQueen M, Court-Brown C. The Disabilities of the Arm, Shoulder and Hand Questionnaire (DASH) can measure the impairment, activity limitations and participation restriction constructs from the International Classification of Functioning, Disability and Health (ICF). BMC Musculoskelet Disord. 2008;9:1-6.

\section{Publisher's Note}

Springer Nature remains neutral with regard to jurisdictional claims in published maps and institutional affiliations.

Ready to submit your research? Choose BMC and benefit from:

- fast, convenient online submission

- thorough peer review by experienced researchers in your field

- rapid publication on acceptance

- support for research data, including large and complex data types

- gold Open Access which fosters wider collaboration and increased citations

- maximum visibility for your research: over $100 \mathrm{M}$ website views per year

At $\mathrm{BMC}$, research is always in progress.

Learn more biomedcentral.com/submissions 